Open Access

\title{
The sialyl-glycolipid stage-specific embryonic antigen 4 marks a subpopulation of chemotherapy-resistant breast cancer cells with mesenchymal features
}

Andrea Aloia', Evgeniya Petrova²,3, Stefan Tomiuk', Ute Bissels', Olivier Déas², Massimo Saini ${ }^{4,5}$, Franziska Maria Zickgraff ${ }^{4,5}$, Steve Wagner ${ }^{4,5}$, Saskia Spaich ${ }^{6}$, Marc Sütterlin ${ }^{6}$, Andreas Schneeweiss ${ }^{7}$, Manuel Reitberger ${ }^{4,5}$, Silvia Rüberg ${ }^{1}$, Bernhard Gerstmayer ${ }^{1}$, David Agorku', Sebastian Knöbel', Annalisa Terranegra ${ }^{8}$, Monica Falleni ${ }^{9}$, Laura Soldati ${ }^{9}$, Martin Ronald Sprick ${ }^{4,5}$, Andreas Trumpp ${ }^{4,5,10}$, Jean-Gabriel Judde ${ }^{2}$, Andreas Bosio ${ }^{1}$, Stefano Cairo ${ }^{2,11^{*}}$ and Olaf Hardt ${ }^{1^{*}}$

\begin{abstract}
Introduction: Chemotherapy resistance resulting in incomplete pathologic response is associated with high risk of metastasis and early relapse in breast cancer. The aim of this study was to identify and evaluate biomarkers of treatment-resistant tumor cells.

Methods: We performed a cell surface marker screen in triple-negative breast cancer patient-derived xenograft models treated with standard care genotoxic chemotherapy. Global expression profiling was used to further characterize the identified treatment-resistant subpopulations.

Results: High expression of sialyl-glycolipid stage-specific embryonic antigen 4 (SSEA4) was found in residual tumor cells surviving chemotherapy and in samples from metastatic patients who relapsed after neoadjuvant chemotherapy. Gene and microRNA (miRNA) expression profiling linked SSEA4 positivity with a mesenchymal phenotype and a deregulation of drug resistance pathways. Functional assays demonstrated a direct link between epithelial-mesenchymal transition (EMT) and SSEA4 expression. Interestingly, SSEA4 expression, EMT, and drug resistance seemed to be regulated posttranscriptionally. Finally, high expression of CMP-N-acetylneuraminate- $\beta$-galactosamide- $\alpha-2,3-$ sialyltransferase 2

(ST3GAL2), the rate-limiting enzyme of SSEA4 synthesis, was found to be associated with poor clinical outcome in breast and ovarian cancer patients treated with chemotherapy.
\end{abstract}

Conclusions: In this study, we identified SSEA4 as highly expressed in a subpopulation of tumor cells resistant to multiple commonly used chemotherapy drugs, as well as ST3GAL2, the rate-limiting enzyme of SSEA4 synthesis, as a predictive marker of poor outcome for breast and ovarian cancer patients undergoing chemotherapy. Both biomarkers and additionally identified regulatory miRNAs may be used to further understand chemoresistance, to stratify patient groups in order to avoid ineffective and painful therapies, and to develop alternative treatment regimens for breast cancer patients.

\footnotetext{
*Correspondence: Stefano.Cairo@xentech.eu; olaf@miltenyibiotec.de

Andrea Aloia and Evgeniya Petrova are co-first authors.

Stefano Cairo and Olaf Hardt are co-senior authors.

${ }^{2}$ XenTech SAS, 4 rue Pierre Fontaine, 91000 Evry, France

'Miltenyi Biotec GmbH, Friedrich-Ebert-Strasse 68, 51429 Bergisch Gladbach,

Germany

Full list of author information is available at the end of the article
}

C Biomed Central

(c) 2015 Aloia et al. Open Access This article is distributed under the terms of the Creative Commons Attribution 4.0 International License (http://creativecommons.org/licenses/by/4.0/), which permits unrestricted use, distribution, and reproduction in any medium, provided you give appropriate credit to the original author(s) and the source, provide a link to the Creative Commons license, and indicate if changes were made. The Creative Commons Public Domain Dedication waiver (http://creativecommons.org/publicdomain/zero/1.0/) applies to the data made available in this article, unless otherwise stated. 


\section{Introduction}

Breast cancer is a heterogeneous disease at molecular and cellular levels. Several subtypes of breast cancer can be defined, depending on molecular marker expression [1], and disease management is currently tailored according to the molecular characteristics of each subtype. Triple-negative breast cancer (TNBC) and the luminal B subtype are characterized as very aggressive and associated with high risk of early relapse and metastasis [2, 3]. Owing to the lack of defined molecular targets for TNBCs and due to the high proliferative rate of TNBCs and luminal B tumors, chemotherapy remains a firstchoice therapeutic option for these two subtypes.

In the neoadjuvant setting, the standard options for TNBC are a combination of doxorubicin/cyclophosphamide (AC) or 5-fluorouracil, epirubicin, and cyclophosphamide followed or not by taxane-containing regimens, or a combination of cyclophosphamide, methotrexate, and 5fluorouracil [4]. The majority of TNBC patients respond initially to neoadjuvant chemotherapy treatment, but only about $20 \%$ reach a pathological complete response, whereas most patients either have lower de novo sensitivity to chemotherapy or develop resistance to chemotherapy [5]. Residual cancer cells persist and initiate tumor recurrence and metastasis within 3 years after chemotherapy in about $40 \%$ of patients [5]. Hence, the development of predictive tests for chemotherapy resistance represents an urgent need that would aid in therapy decision-making.

Many studies have been reported that provide data about the molecular basis of human breast cancers. However, the high degree of heterogeneity within the tumor, as well as the varying response to chemotherapy of cellular subclones, makes the interpretation of these molecular profiles difficult [6]. In particular, if biomarkers correlating with treatment outcome are expressed only in subpopulations of tumor cells, the analysis of bulk tumor material might lack sensitivity. Furthermore, tumor cell subpopulations can change their phenotype and gene expression profile to escape chemotherapy by means of epithelial-mesenchymal transition (EMT), upregulation of multidrug resistance transporters, and modulation of key signaling pathways $[7,8]$. Thus, it is crucial to analyze marker expression patterns at different time points during chemotherapy, when the tumor cells are either developing drug resistance or de novo resistant subpopulations are selected.

In the present study, we performed a flow cytometrybased screen of cell surface markers in patient-derived xenografts (PDXs) of TNBC tumor samples during AC chemotherapy. PDXs exhibit morphology, molecular characteristics, and drug response profiles similar to those of the original patient tumors [9] and thereby represent a reliable model and a reproducible source for human tumor cells needed for detailed analyses of tumor cell subpopulations over time $[9,10]$.
We observed that a high percentage of residual tumor cells surviving chemotherapy at surgical ablation express the sialyl-glycolipid stage-specific embryonic antigen 4 (SSEA4). Molecular profiling revealed mesenchymal traits as well as upregulation of genes involved in multidrug resistance in SSEA4-positive compared with SSEA4-negative tumor cell subpopulations. Elevated expression of CMP- $N$-acetylneuraminate- $\beta$-galactosamide$\alpha$-2,3-sialyltransferase 2 (ST3GAL2), the enzyme catalyzing the last step of SSEA4 synthesis, is associated with poor prognosis in breast cancers treated with chemotherapy. This predictive value was also confirmed in a cohort of ovarian carcinoma. Thus, we propose SSEA4 as a novel marker for EMT-associated chemotherapy resistance and ST3GAL2 expression as a predictive marker for tumor resistance to chemotherapy.

\section{Methods}

A detailed description of materials and methods can be found in additional file 1.

\section{Primary tissue material and xenotransplantation}

Human breast cancer xenografts ( $\mathrm{HBCx}$ ) were established from patient's primary tumor surgical specimens by grafting tumor fragments into the interscapular fat pad and maintained through in vivo passages as previously described [9]. All experiments were performed in accordance with French legislation concerning the protection of laboratory animals and in accordance with a currently valid license issued by the French Ministry for Agriculture and Fisheries for experiments on vertebrate animals. The ethics committee was organized according to the pertinent French legislation and was approved by the French Ministry of Research under number CE 51.

Primary serous ovarian carcinoma cell lines were established by transplantation of primary tumor specimen or tumor cells directly isolated from ascites or pleural effusion samples. Human tumors were injected intraperitoneally into NOD.Cg-Prkd $c^{\text {scid }} I l 2 r g^{t m 1 W_{j l}}$ mice. Engrafted first passage xenografts were dissociated into single cells and maintained under serum-free culture conditions. Animal care and all procedures were carried out according to German legal regulations and were previously approved by the governmental review board of the state of Baden-Wuerttemberg (Regierungspräsidium Karlsruhe authorization number G17/12).

This study was performed with human tissue samples obtained from patients admitted to the University Clinic Mannheim Department of Gynecology. The study was approved by the ethics committee of the University of Heidelberg-Mannheim (case number 2011-380N-MA) and conducted in accordance with the Declaration of Helsinki. Written informed consent was obtained from all patients. In addition, primary patient samples of clear cell renal cell 
carcinoma (RCC) were obtained from the Department of Health Sciences at the University of Milan. All samples were collected according to the regulations for the use of primary material according to "doc. web n. 1878276" (Pubblicato sulla Gazzetta Ufficiale n. 72; 26 Mar 2012).

\section{Cell lines used}

The epithelial breast cell line MCF 10A was purchased from the American Type Culture Collection (ATCC ${ }^{\circ}$ CRL-10317 ${ }^{\mathrm{m}}$; ATCC, Manassas, VA, USA). The HBCx17 and $\mathrm{HBCx}-39$ cell lines were primary cells derived for the respective $\mathrm{HBCx}$ tumors at XenTech SAS (Evry, France). The OC-12, OC-14, OC-15, OC-18, OC-19, and OC-20 cell lines were primary cells derived for the respective ovarian cancer xenograft tumors at HI-STEM gGmbH (Heidelberg, Germany).

\section{Chemotherapeutic treatment}

Doxorubicin (ADRIBLASTINA ${ }^{\bullet}$ RD; Pfizer, New York, NY, USA) and cyclophosphamide (ENDOXAN ${ }^{\circ}$; Baxter Healthcare, Deerfield, IL, USA) solutions were administered on the same day via intraperitoneal injection at a dose of $2 \mathrm{mg} / \mathrm{kg}$ (doxorubicin) and $100 \mathrm{mg} / \mathrm{kg}$ (cyclophosphamide). To obtain a complete response for models $\mathrm{HBCx}-17$ and $\mathrm{HBCx}-6$, the same dose of AC chemotherapy was applied a second time, 3 weeks after the first injection. AC chemotherapy was applied to 68 mice of tumor graft model $\mathrm{HBCx}-17,32$ mice of $\mathrm{HBCx}-10,35$ mice of $\mathrm{HBCx}-6$, and 30 mice of $\mathrm{HBCx}-14$ model, not including the control group.

\section{Flow cytometry-based analysis}

Tumor tissue was dissociated into a single-cell suspension using the human Tumor Dissociation Kit in combination with the gentleMACS Octo Dissociator (both from Miltenyi Biotec, Bergisch Gladbach, Germany) according to the manufacturer's instructions. Cells were stained with the indicated antibodies (Additional file 2: Table S1) according to the manufacturer's instructions and analyzed using the MACSQuant ${ }^{\mathrm{Tm}}$ Analyzer (Miltenyi Biotec) (Additional file 3: Figure S1). In the cases of SSEA4, TRA-1-60, and TRA-1-81, recombinant antibodies were available and were used because of their superior characteristics $[11,12]$. The specificity of all recombinant antibodies was validated and compared with conventional clones. In the case of SSEA4, identical specificity for antibodies derived from clone MC-813-70 and clone REA101 was proven by cross- blocking experiments, which showed that either antibody specifically blocks binding of the alternative one, suggesting that both antibodies bind the same epitope on the target structure SSEA4 (Additional file 4: Figure S2).
Isolation of SSEA4-positive and SSEA4-negative tumor cell subpopulations

SSEA4-positive and SSEA4-negative tumor cell subpopulations were isolated by magnetic activated cell sorting (MACS ${ }^{\circledast}$ Technology; Miltenyi Biotec). After dissociation and depletion of mouse cells using the Mouse Cell Depletion Kit (Miltenyi Biotec), the cells were labeled with SSEA4-phycoerythrin (Miltenyi Biotec) followed by anti-phycoerythrin MicroBeads (Miltenyi Biotec) and separated using MS and LD columns (Miltenyi Biotec). For microarray analysis, cells were pelleted and lysed in QIAzol $^{\circledR}$ (QIAGEN, Hilden, Germany).

\section{EMT induction}

To induce EMT, the epithelial breast cell line MCF 10A was treated with transforming growth factor (TGF)- $\beta 1$ (Miltenyi Biotec) at concentrations of 10 and $20 \mathrm{ng} / \mathrm{ml}$. EMT markers such as epithelial cell adhesion molecule (EpCAM), E-cadherin, vimentin, and fibronectin served as indicators of EMT induction efficiency.

\section{Microarray hybridization and data analysis}

Messenger RNA (mRNA) and microRNA (miRNA) expression profiling was performed using Agilent microarrays (Agilent Technologies, Santa Clara, CA, USA) according to the manufacturer's instructions.

The mRNA and miRNA data discussed in this publication have been deposited in the National Center for Biotechnology Information Gene Expression Omnibus (GEO) $[13,14]$ and are accessible under GEO series accession number [GEO:GSE57705] (http://www.ncbi.nlm.nih.gov/ geo/query/acc.cgi?acc=GSE57705).

\section{Clinical data analysis}

To evaluate the prognostic value of candidate genes, the publicly available Kaplan-Meier Plotter (http://kmplot.com/ analysis/) was used. The Kaplan-Meier survival plots of the two patient cohorts were compared using logrank test with hazard ratios (HRs) and $95 \%$ confidence intervals $[15,16]$.

\section{In vitro cytotoxicity assays}

Cells were seeded in 96-well flat-bottom plates. After 2 days at $37{ }^{\circ} \mathrm{C}$ and $5 \% \mathrm{CO}_{2}$, the cells were treated with different standard chemotherapy drugs. To obtain a dose-response curve, each drug was tested at serial concentrations. Cell viability was analyzed $72 \mathrm{~h}$ after addition of drugs using the CellTiter-Glo ${ }^{\circ}$ Luminescent Cell Viability Assay Kit (Promega, Madison, WI, USA) according to the manufacturer's instructions. Luciferase activity was measured on a luminometer (PerkinElmer ${ }^{\circledR}$ EnVision $^{\mathrm{TM}}$; PerkinElmer, Waltham, MA, USA). 


\section{Results}

The expression of 23 cell surface markers in breast cancer PDXs is affected by chemotherapy

An antibody screening based on a library of 45 antibodies directed against surface epitopes including published stem cell and/or cancer stem cell markers (Additional file 2: Table S1) was performed to identify novel biomarkers for breast cancer cell subpopulations resistant to chemotherapeutic treatment. For the initial screening, 50-100 xenografted mice were used for each of four independent TNBC PDXs [9]. When tumor volumes reached $150-350 \mathrm{~mm}^{3}$ (pretreatment stage), mice were treated with an AC combination according to the standard of care. After tumor shrinking to volumes of 14-63 $\mathrm{mm}^{3}$, the nodules were removed (residual tumor stage). Tumors from untreated mice were removed (untreated stage) to serve as direct controls. A group of animals with residual tumors were kept until the disease recurred (regrowth stage) (Fig. 1a). All tumors removed at any time point were dissociated and analyzed by flow cytometry. No significant differences concerning marker expression were found between the pretreatment and untreated stages, excluding size-dependent marker regulation, whereas the expression of 10 markers decreased (Fig. 1c) and the expression of 13 markers increased (Fig. 1d) during chemotherapy. Eighty-seven percent (20 of 23) of these markers returned to an expression level similar to the untreated stage upon tumor regrowth (Fig. 1c and d). CD44 and CD133, which have been described to correlate with a cancer stem cell and drug resistance phenotype [17], showed enrichment in only one of the four models. This is possibly due to the tumor heterogeneity that characterizes patients' tumors, which was here well recapitulated by the use of PDX models (Fig. 1d). However, we identified a distinct subpopulation of tumor cells expressing SSEA4 that was strongly enriched during chemotherapeutic treatment in all tumor models analyzed. Independent replicates of three tumor models confirmed significant enrichment in the number of SSEA4-positive cells in residual tumors upon AC treatment in all analyzed tumors $(p<0.001, n=8$ for each tumor model) (Fig. 1e). This result was further confirmed by immunohistochemistry (Fig. 1b). Similarly to other markers, the amount of SSEA4-positive cells decreased back to pretreatment levels in three of four tumor models after the treatment was stopped.

To further address the correlation between CD24, CD44, and SSEA4 expression, we performed containing of these markers on residual tumor nodules after AC chemotherapy and on untreated tumors of three independent models (Additional file 5: Figure S3). We did not observe a significant enrichment of the CD24 $4^{\text {low }}$ / $\mathrm{CD} 44^{\text {high }}$ subpopulation in any of the models. In model $\mathrm{HBCx}-6$, there was a subpopulation of SSEA4 $4^{+} / \mathrm{CD} 44^{+}$ cells that was enriched by about twofold; however, the SSEA4 ${ }^{+} / \mathrm{CD}_{4} 4^{-}$fraction was enriched to the same degree. In models $\mathrm{HBCx}-10$ and $\mathrm{HBCx}-14, \mathrm{CD} 44$ and SSEA4 expression was observed on separate subpopulations. We concluded that SSEA4 expression is an indicator of treatment-resistant cells that overcomes the heterogeneity observed for the (cancer) stem cell markers in the tumor models used in this study.

\section{SSEA4 is a marker for de novo resistance to chemotherapy treatment}

In one PDX model, we observed tumors with variable sensitivity to the $\mathrm{AC}$ treatment and noticed that the amount of SSEA4-positive cells correlated with these differences. Nineteen tumors showed reduced sensitivity to AC treatment (tumor volume $>100 \mathrm{~mm}^{3}$ as maximal regression), and one tumor did not respond to the treatment at all (no tumor shrinkage during treatment). The percentages of SSEA4-positive cells were $36.8 \%$ in sensitive tumors, $42.2 \%$ in tumors with reduced sensitivity, and $98.5 \%$ in the fully resistant tumor (data not shown). Therefore, we compared tumors from PDX models that are sensitive $(n=6)$ or de novo resistant $(n=4)$ to $\mathrm{AC}$ treatment. Three of four resistant tumor models showed higher percentages of SSEA4-positive cells than the six sensitive tumors (Additional file 6: Figure S4).

To provide further evidence of resistance to drug toxicity, we established primary PDX-derived ex vivo cell lines from tumors containing different amounts of SSEA4-positive cells and treated them with chemotherapeutic drugs in vitro. One of the cell lines derived from model HBCx-17 showed reproducible growth as an adherent culture and as a suspension culture, with cells growing in suspension showing higher SSEA4 expression than the adherent cells (Fig. 2a). In cytotoxicity assays with seven commonly used drugs (Fig. 2b-f and data not shown), the suspension cells showed higher halfmaximal inhibitory concentration values than the adherent cells for the DNA synthesis and transcription inhibitors cisplatin, mafosfamide, 5-fluorouracil, and doxorubicin, indicating an increased resistance, but not for the topoisomerase inhibitors etoposide, topotecan, and irinotecan. To provide a dose-escalating reproduction of our in vivo experiment, we treated an adherent cell line derived from model $\mathrm{HBCx}-17$, containing about $15 \%$ SSEA4-positive cells in the steady state, and analyzed the phenotype of the cells surviving the treatment upon administration of increasing drug dosages. In every case, the surviving population showed a significantly higher fraction of SSEA4-positive cells (Fig. 2g-i), while the absolute number of SSEA4-positive cells was not significantly increased.

Next, we evaluated possible differences in the tumorigenic capacity of SSEA4-positive and SSEA4-negative 


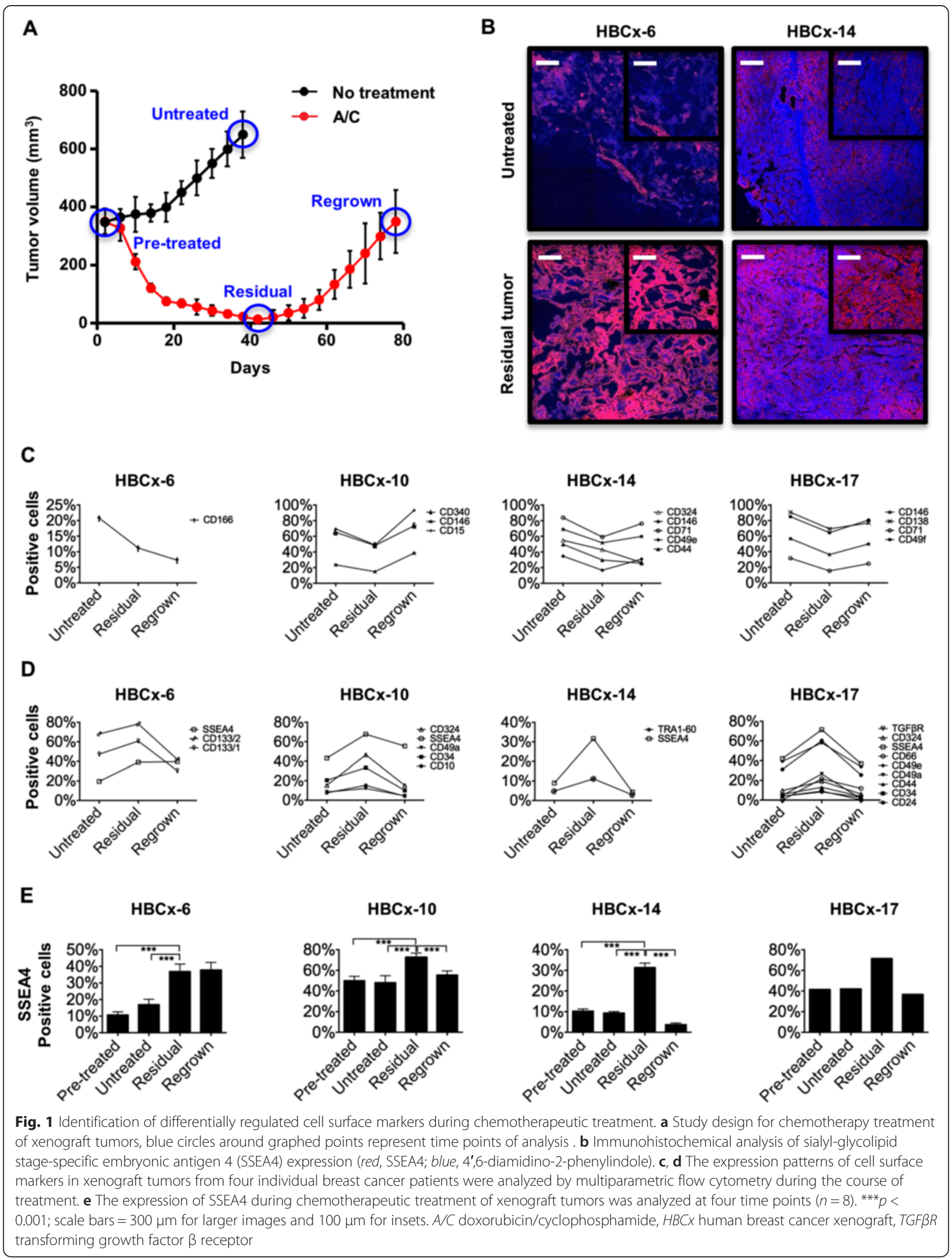




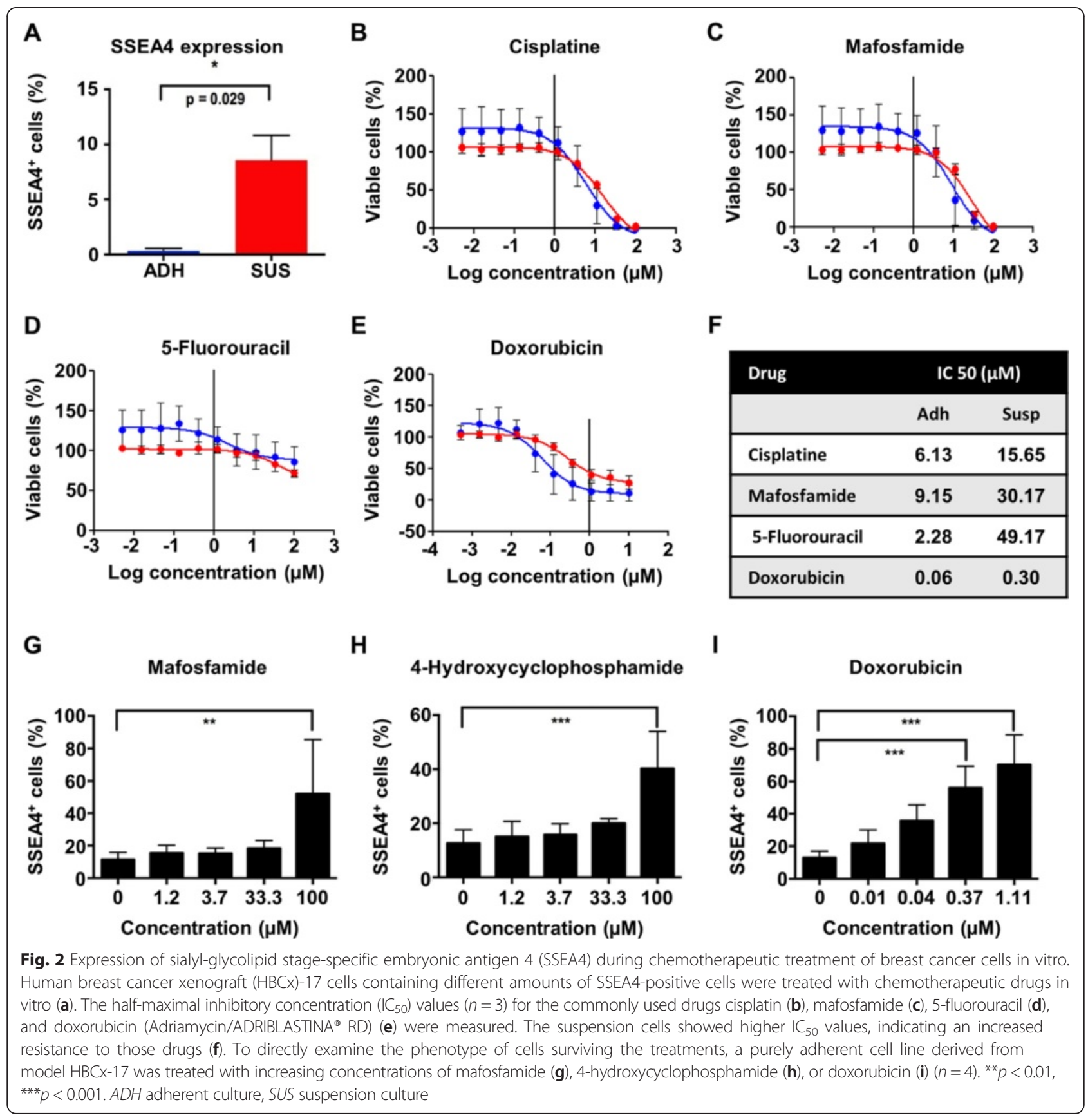

subpopulations in model $\mathrm{HBCx}-14$, which shows about $10 \%$ SSEA4-positive tumor cells when growing without any treatment and a significant upregulation under genotoxic stress. To do so, $10^{5}$ freshly dissociated SSEA4positive or SSEA4-negative cells were injected into two groups of eight mice each. Although a trend toward a faster tumor initiation was observed when we grafted the SSEA4-positive tumor subpopulation, both fractions were able to generate tumors, indicating that the positive fraction only has an initial growth advantage (Additional file 7: Figure S5a). Furthermore, we analyzed the expression of SSEA4 in tumors that arose from both subpopulations. Whereas the fraction of SSEA4-expressing cells in tumors that originated from the SSEA4-negative subpopulation recovered to around $5 \%$, the one from tumors that originated from the SSEA4-positive subpopulation was reduced to about $10 \%$ following the growth phase in vivo (Additional file 7: Figure S5b). The recovery of the original SSEA4positive versus SSEA4-negative ratio was also reflected by the observation that when freshly dissociated SSEA4-positive and SSEA4-negative cells were placed 
in culture separately, they restored the original positive versus negative ratio within approximately 6 days (data not shown), which correlated well with the observation of SSEA4 downregulation during disease relapse after chemotherapy.

\section{SSEA4 expression is found in metastatic cells that survived genotoxic chemotherapy}

Local or metastatic breast cancer relapse may occur many years after surgery, despite a short-term response to neoadjuvant chemotherapy [18] or a failure of adjuvant chemotherapy. To investigate SSEA4 expression in metastatic relapse, we conducted a retrospective case history study using PDXs derived from metastatic specimens. These models were derived from confirmed M1stage patients through collection of liquid biopsies, either peripheral circulating tumor cells (CTCs) or infiltrating CTCs obtained from serous effusions [19]. Robust SSEA4 expression was exclusively found in PDXs whose donors were previously treated with specific neoadjuvant chemotherapy formulations, including the AC combination and other genotoxic drugs with analogous modes of action, such as epirubicin (Fig. 3). Conversely, metastatic patients who were administered taxols (paclitaxel, docetaxel) or antimetabolites (capecitabine, 5-fluorouracil) as adjuvant therapy, but who had no genotoxic treatment before sample collection (Fig. 3 and Additional file 8: Table S2 for patient history), displayed little or no expression of SSEA4 on their matched PDXs (Fig. 3).

Molecular analysis of SSEA4-positive and SSEA4-negative subpopulations

We performed microarray-based global mRNA and miRNA expression profiling on SSEA4-positive and SSEA4-negative cells using three independent TNBC models: HBCx-6 ( $n=7$ tumors), HBCx-10 ( $n=5$ tumors), and HBCx-14 ( $n=9$ tumors). To avoid a bias by cells of murine origin [20], all mouse cells were depleted after tumor dissociation (Fig. 4a) and SSEA4-positive cells were labeled and magnetically separated from the negative fraction (Fig. 4b) before expression profiling.

Transcripts with significantly increased (240 genes, $p<0.05, \geq 1.5$-fold, Additional file 9: Table S3) or decreased (182 genes, $p<0.05$, greater than or equal to -1.5-fold, Additional file 9: Table S3) expression in SSEA4-positive compared with SSEA4-negative cells were subjected to a term enrichment analysis based on Gene Ontology categories [21]. In the SSEA4-positive fractions, we found a strong overrepresentation of genes linked to the TGF- $\beta$ and epidermal growth factor signaling pathways as well as genes involved in cell adhesion and migration and in regulation of apoptosis, proliferation, and differentiation (Additional file 9: Table S3). Among the genes upregulated across all tumor models, seven functional groups involved in cellular import and export,

A

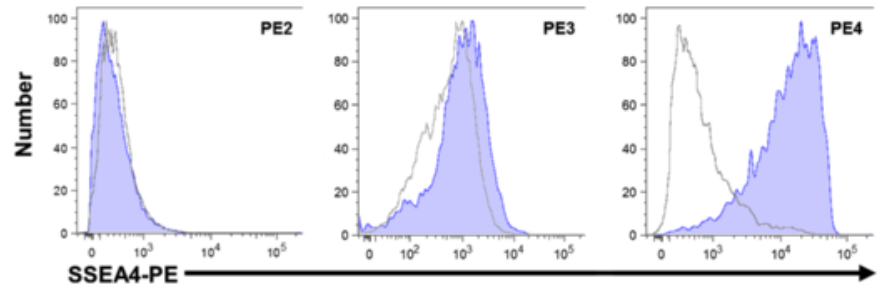

B

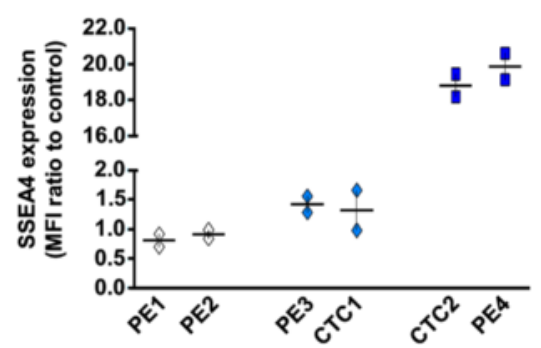

C

\begin{tabular}{|cccc|}
\hline ID & Origin & Neoadj. CT & Adjuvant CT \\
\hline PE1 & PE & no & Non-genotoxic \\
\hline PE2 & PE & no & Non-genotoxic \\
\hline PE3 & Ascites & no & Genotoxic \\
\hline CTC1 & CTC & no & Genotoxic \\
\hline CTC2 & CTC & $\begin{array}{c}\text { Genotoxic } \\
\text { (Epirubicin) }\end{array}$ & Non-genotoxic \\
\hline PE4 & PE & Genotoxic (A/C) & Non-genotoxic \\
\hline
\end{tabular}

Fig. 3 Sialyl-glycolipid stage-specific embryonic antigen 4 (SSEA4) expression is found in metastatic cells that survived genotoxic chemotherapy. a The surface expression of SSEA4 on primary cells from patient-matched xenografts (passages 1-3) was evaluated. Each xenograft was stained with an isotype control (gray histogram) or with an SSEA4 antibody (blue histogram). b Quantification of SSEA4 expression on the cell surface of six xenografts obtained from metastatic pleural effusions (PE) or circulating tumor cells (CTCs) from M1-stage metastatic breast cancer patients. The median fluorescence intensity (MFI) ratio is calculated as the MFI of SSEA4-stained cells divided by the MFI of the isotype control-stained cells. c Schematic overview of each metastatic breast cancer patient's treatment characteristics. CT chemotherapy) 


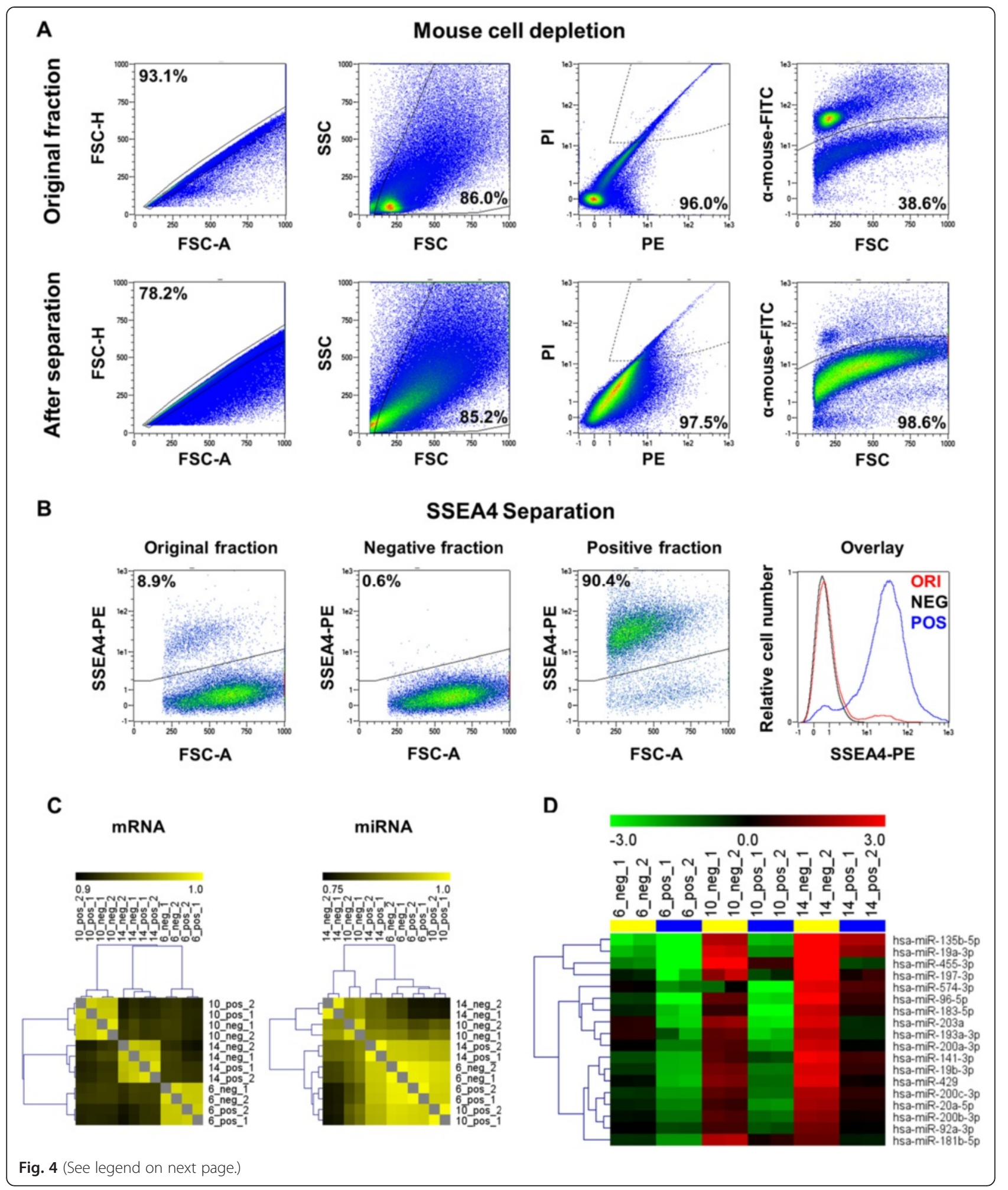


(See figure on previous page.)

Fig. 4 Isolation and molecular analysis of sialyl-glycolipid stage-specific embryonic antigen 4 (SSEA4)-positive and SSEA4-negative tumor subpopulations. $\mathbf{a}$ and $\mathbf{b}$ Flow cytometric analysis of cells before and after cell sorting. c Correlation matrices showing the relationship of mRNA- and miRNA-based gene expression profiles in all experiments. Correlation coefficients are indicated by their color from 0.9 (black) to 1.0 (yellow) for mRNA-based and from 0.75 (black) to 1.0 (yellow) for miRNA-based clustering. $\mathbf{d}$ Cluster analysis of miRNAs identified as differentially expressed by discriminatory gene analysis in combination with a paired $t$ test $(p<0.05)$ of the SSEA4-positive versus SSEA4-negative tumor subpopulation. Resulting genes were grouped by similarities in gene expression patterns using hierarchical clustering (Pearson correlation, average linkage). Levels of $\log _{2}$-transformed expression ratios are indicated from -3 (green) to 3 (red). FITC fluorescein isothiocyanate, FSC forward scatter FSC-A forward scatter area, FSC-H forward scatter height, miRNA microRNA, MRNA messenger RNA, PE pleural effusions, PI propidium iodide, SSC side scatter

response to toxins and oxidative stress were significantly enriched (Additional file 9: Table S3). In particular, members of the solute carrier (SLC) and multidrug resistance ATP-binding cassette transporter families were significantly upregulated (Additional file 10: Figure S6B).

Also, among the differentially expressed transcripts, we identified a substantial number of genes involved in EMT. Epithelial markers such as cytokeratin 19, CLDN1, CLDN3, and CLDN4 showed lower expression in SSEA4-positive cells, whereas mesenchymal indicators such as fibronectin, vitronectin, $Z E B 1$, and $Z E B 2$ were upregulated (Additional file 10: Figure S6 A). In contrast, published stem cell markers were not consistently regulated among the SSEA4-positive and SSEA4-negative cell fractions (Additional file 10: Figure S6 C). As SSEA4 is a glycolipid epitope, its expression cannot be monitored directly by transcriptome profiling. SSEA3, the direct precursor of SSEA4, showed no increased signal intensity in SSEA4-positive cells when measured with anti-SSEA3 antibody in all analyzed models (data not shown). This indicates that SSEA4 enrichment regulation may be due to increased SSEA3-to-SSEA4 conversion or to increased SSEA4 catabolism in SSEA4-negative cells. However, no difference was observed in the expression of genes coding for enzymes involved in degradation of SSEA4 or of ST3GAL2, the enzyme catalyzing this final step of SSEA4 synthesis [22]. In expression analysis of the miRNA data set, we identified 166 miRNAs more than twofold overrepresented and 68 miRNAs more than twofold underrepresented in SSEA4-positive versus SSEA4-negative cells among all analyzed tumor models (Additional file 9: Table S3). Hierarchical clustering of the Pearson correlation coefficients of our mRNA and miRNA expression datasets showed a higher correlation of SSEA4-positive versus SSEA4-negative phenotype in the miRNA rather than mRNA data set (Fig. 4c). No miRNAs were significantly upregulated, and 18 miRNAs were significantly downregulated $(p<0.05$ by paired $t$ test), in SSEA4positive cells (Fig. 4d and Additional file 9: Table S3). These results were independently validated by using a flow cytometry-based 39-plex miRNA assay (Additional file 11: Figure S7) for direct measurement of miRNAs without prior amplification. To determine putative mRNA targets for these candidates, we used six different computational target prediction tools and considered only targets that were predicted by at least two target prediction algorithms. On the basis of this analysis, 5 of the 18 miRNAs downregulated in SSEA4-positive cells (miR-96-5p, miR200b-3p, miR-200c-3p, miR-429, and miR-92a-3p) are predicted to target ST3GAL2, suggesting that SSEA4 expression is regulated posttranscriptionally. Furthermore, 12 of the 18 downregulated miRNAs are known to target key mesenchymal regulator and indicator genes such as ZEB1, ZEB2, fibronectin 1, Snail1, Snail2, and Twist (Additional file 12: Figure S8 and Additional file 9: Table S3).

\section{SSEA4 expression is regulated by ST3GAL2}

To provide functional confirmation of the role of ST3GAL2 in the regulation of SSEA4 expression in PDX samples, we knocked down ST3GAL2 by small interfering RNA (siRNA) in HBCx-39 cells, which show high levels of SSEA4 expression, and analyzed the expression of SSEA4. A significant decrease of SSEA4 expression was observed upon ST3GAL2 expression inhibition $(p<0.001, n=6)$ (Fig. 5a and b). Knockdown of CD133 cell surface expression was used as a positive control for siRNA targeting, and it had no impact on SSEA4 expression. To provide further evidence of the relationship between ST3GAL2 and SSEA4, we compared ST3GAL2 mRNA levels with SSEA4 cell surface levels on nine different tumor models, which showed significant positive correlation $(p<0.002$, Fig. $5 c)$.

\section{Epithelial-mesenchymal transition induces SSEA4 expression}

As EMT has already been correlated with drug resistance [23], we wanted to examine if SSEA4 expression was increased after EMT induction. Upon treatment of the epithelial breast cell line MCF 10A with TGF- $\beta 1$, almost all cells changed their morphology from an epithelial to an elongated fibroblastic shape (Fig. 6a). As expected [24], epithelial markers such as EpCAM and E-cadherin were downregulated, while mesenchymal markers such as vimentin and fibronectin were upregulated (Fig. 6a and data not shown). Upon EMT 

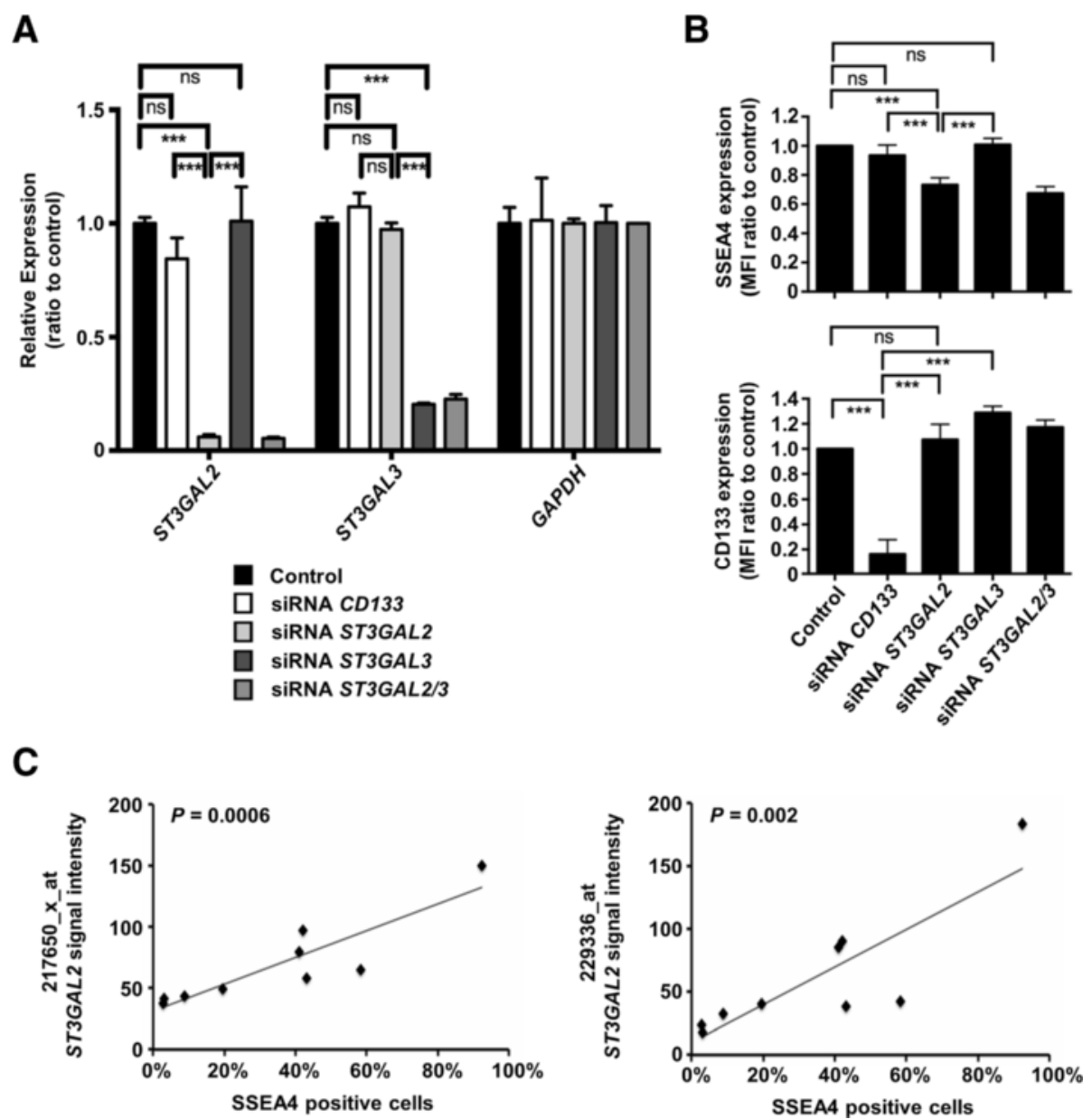

Fig. 5 Sialyl-glycolipid stage-specific embryonic antigen 4 (SSEA4) expression is regulated by CMP-N-acetylneuraminate- $\beta$-galactosamide-a2,3-sialyltransferase 2 (ST3GAL2). a Small interfering RNA (siRNA)-mediated knockdown of ST3GAL2 and ST3GAL3 significantly reduced the expression of their respective target messenger RNAs (mRNAs), but not the housekeeping gene GAPDH, as measured by quantitative real-time polymerase chain reaction. Each bar represents the expression intensity normalized to the Lipofectamine reagent (Life Technologies, Carlsbad, CA, USA)-only control. b Knockdown of ST3GAL2 significantly reduced the expression of SSEA4, whereas targeting of CD133 or the close paralog ST3GAL3 did not result in a significant change of SSEA4 expression. Knockdown of CD133 expression by the respective siRNA was used as a positive control of direct targeting. Each bar represents the mean fluorescence intensity (MFI) normalized to the Lipofectamine-only control. c Expression of ST3GAL2 mRNA was measured by two Affymetrix ${ }^{\oplus}$ (Affymetrix, Santa Clara, CA, USA) probes, 217650_x_at and 229336_at, in nine tumor models and plotted against the frequency of SSEA4-positive cells as measured by flow cytometry in the respective models, which showed a significant positive correlation. ${ }^{* *} p<0.001$; ns = not significant; $n=6$

induction, the SSEA4-positive fraction increased more than threefold, hence proving a causal correlation between the transition toward a mesenchymal phenotype and SSEA4 expression (Fig. 6b).

\section{ST3GAL2 is a highly significant predictive and prognostic marker in breast cancer patients}

Given the functional connection between SSEA4 and ST3GAL2, we evaluated the clinical value of ST3GAL2 in a large, publicly available clinical microarray database of breast tumors from 2977 patients [15]. Highly significant differences $(p<0.01)$ trending toward a poorer outcome for patients expressing higher levels of ST3GAL2 within the estrogen receptor-negative $\left(\mathrm{ER}^{-}\right)$or $\mathrm{ER}^{-} /$progesterone receptor-negative $\left(\mathrm{PR}^{-}\right)$ subset of patients were found, independent of the treatment (Fig. 7a). When we focused on patients treated with chemotherapy, we observed a highly significant reduction of relapse-free survival independent from the tumor subtype $(p<0.01, \mathrm{HR} 1.91)$ in $\mathrm{ER}^{-}$ patients $(p<0.01, \mathrm{HR} 2.97)$ and in $\mathrm{ER}^{-} / \mathrm{PR}^{-}$patients $(p<0.01$, HR 3.08) among patients expressing high levels of ST3GAL2 (Fig. 7b). When we applied distant metastasis-free survival as an endpoint, we found that patients expressing high levels of ST3GAL2 had a worse outcome, confirming the involvement of SSEA4-positive 
A
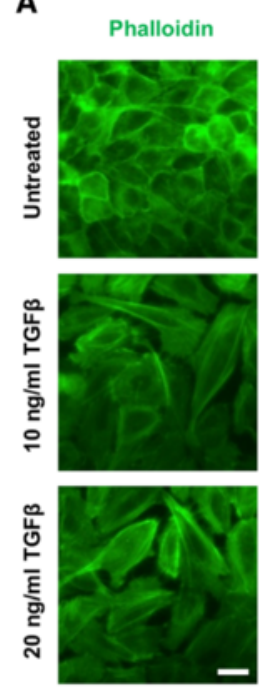
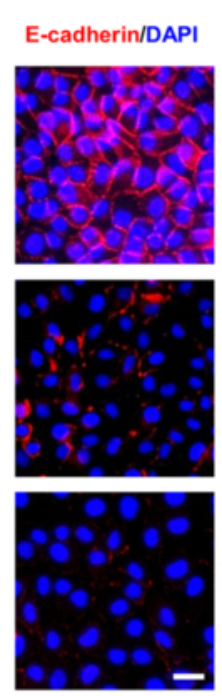
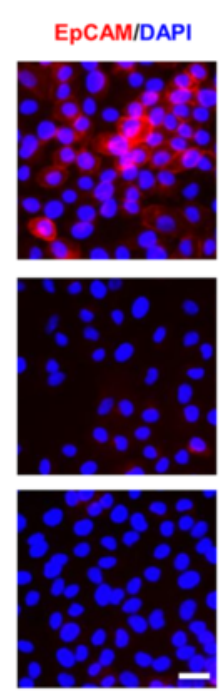
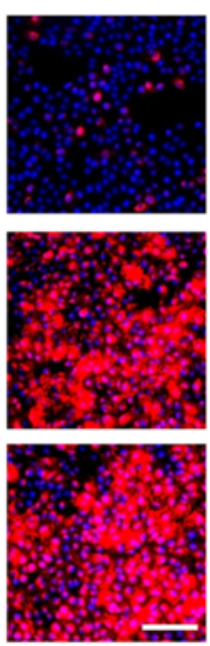

B

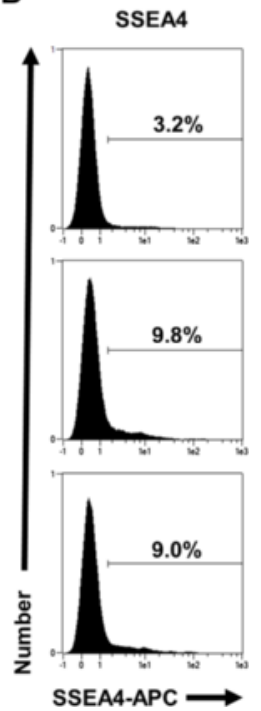

Fig. 6 Epithelial-mesenchymal transition (EMT) induces sialyl-glycolipid stage-specific embryonic antigen 4 (SSEA4) expression. a The expression of SSEA4 was evaluated upon EMT induction. Upon treatment, almost all cells changed their morphology from an epithelial to an elongated fibroblastic shape. F-actin was stained with phalloidin to visualize the cytoskeleton architecture. The epithelial markers E-cadherin and epithelial cell adhesion molecule (EpCAM) were downregulated, whereas the mesenchymal marker fibronectin was upregulated, upon stimulation. Scale bar $=10 \mu \mathrm{m}$ (first three columns at left) and $200 \mu \mathrm{m}$ (right column). b The fraction of SSEA4-positive cells was increased upon EMT induction as evaluated by flow cytometry. APC allophycocyanin, DAPI 4',6-diamidino-2-phenylindole, TGF $\beta$ transforming growth factor $\beta$

cells in metastasis formation (Fig. 7c), as observed in the case of metastasis-derived PDXs.

\section{SSEA4 and ST3GAL2 expression predict chemoresistance and are associated with patient outcomes in other carcinomas}

To further evaluate SSEA4 expression as a marker of intrinsic tumor cell resistance to chemotherapy, we tested SSEA4 expression in samples from primary clear cell RCC, a tumor entity known to be de novo resistant to chemotherapy in more than $95 \%$ of patients [25] and from late ovarian cancer, an aggressive disease whose treatment relies on chemotherapy as the only therapeutic option [26]. Primary clear cell RCC as well as healthy kidney tissues from the same patients were analyzed for SSEA4 expression. In all of the analyzed patients $(n=3)$, an elevated number of SSEA4-positive cells was observed in the tumor tissue (Additional file 13: Figure S9). Similarly, when primary cells derived from serous ovarian carcinoma specimens were analyzed for expression of SSEA4, three of three samples from tumors that previously received genotoxic treatment showed SSEA4 expression above $10 \%$, whereas only one primary cell line matched to treatment-naive patients showed SSEA4 expression above 10 \% (Fig. 8a-c). Analysis of mesenchyme-specific (FN1, SNAI1, ZEB2) and epithelial $(C L D N 3)$ genes by quantitative real-time polymerase chain reaction revealed that ovarian cancer primary cells with high SSEA4 expression showed higher expression of mesenchyme-specific genes and lower expression of the epithelium-specific ones (Fig. 8a and b).

In light of these results, we evaluated whether ST3GAL2 expression could be associated with patient prognosis in ovarian cancer. Using the whole dataset of ovarian tumors from 1464 patients [16], a significant difference trending toward a worse clinical outcome was observed with respect to progressionfree survival $(p<0.05)$ as well as postprogression survival $(p<0.05)$ (Fig. 8e). When we assessed patients treated with chemotherapy, the level of significance was also strongly increased for both endpoints $(p<0.01)$ (Fig. 8f).

\section{Discussion}

In the present study, we identified a novel subpopulation of chemotherapy-resistant tumor cells defined by the expression of SSEA4, the highest-order glycosphingolipid (GSL) in the globo series synthetic pathway starting from glucosylceramide. Enrichment of SSEA4-positive cells in residual tumors of all the tumor models used in this study suggests that this pluripotency marker overcomes the heterogeneity shown by many cancer stem markers analyzed (e.g., CD44, CD133, CD117, CD271, ABCG2) [27-29] that were found to be enriched only in single models. A large body of evidence indicates that upregulation of ceramide glycosylation by 


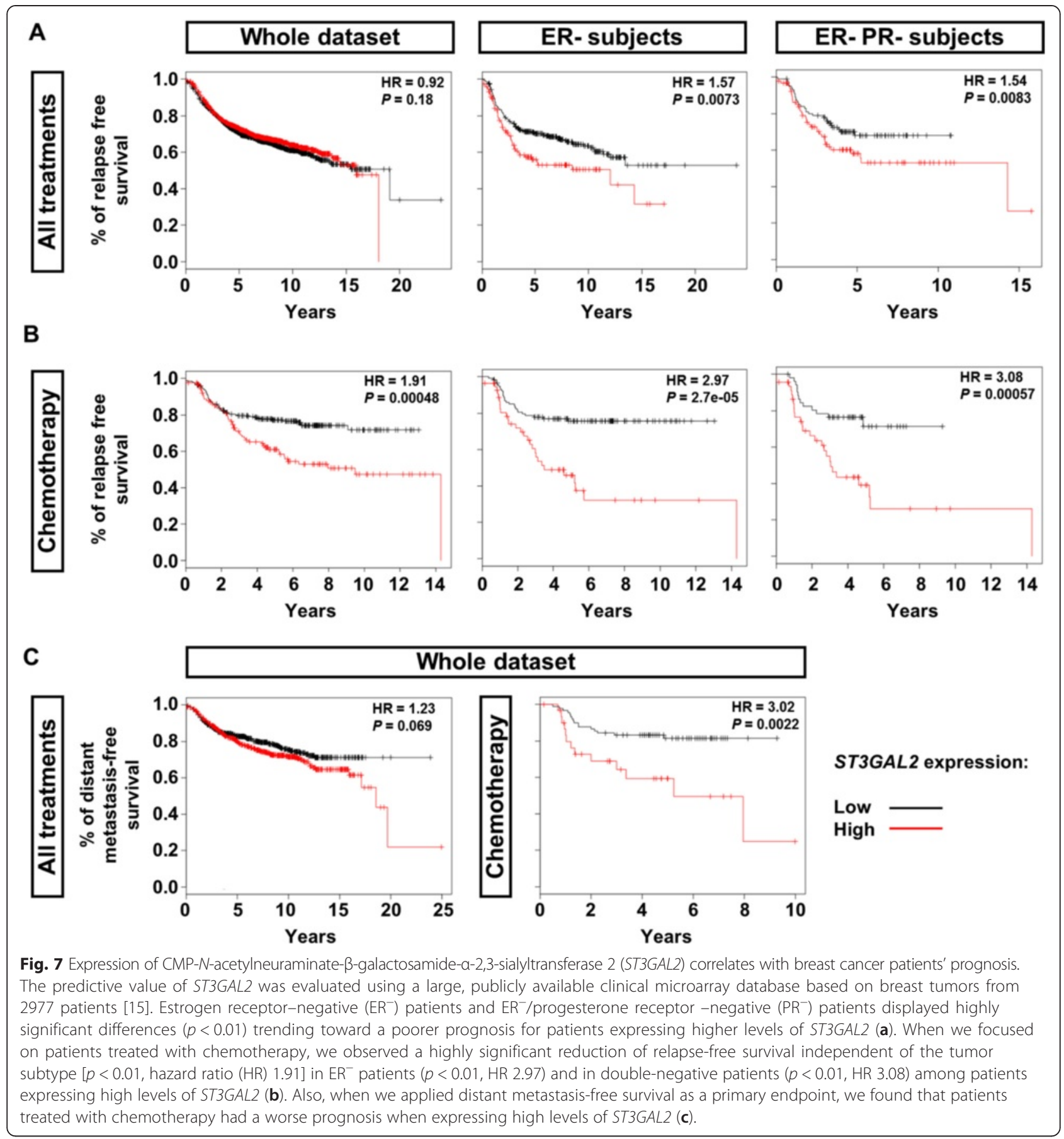

glucosylceramide synthase, through its ability to increase levels of high-order GSLs, contributes to acquisition of drug resistance in cancer cells [30]. In our experimental model, the fraction of SSEA4-positive cells returned to pretreatment levels in the majority of PDXs upon tumor regrowth, indicating that this subpopulation has no general growth advantage. Indeed, this behavior was proposed for other subpopulations of drug-resistant cells
[31]. Accordingly, we did not observe a significant difference in tumorigenic capacity among SSEA4-positive or SSEA4-negative subpopulations. Similar observations have been made in non-small cell lung cancer, where residual tumor cells driving disease relapse after chemotherapy appear to be in an EMT state but do not show any enrichment of cancer stem cell marker-positive cells or enhanced tumor-initiating capacity [23]. Given that 


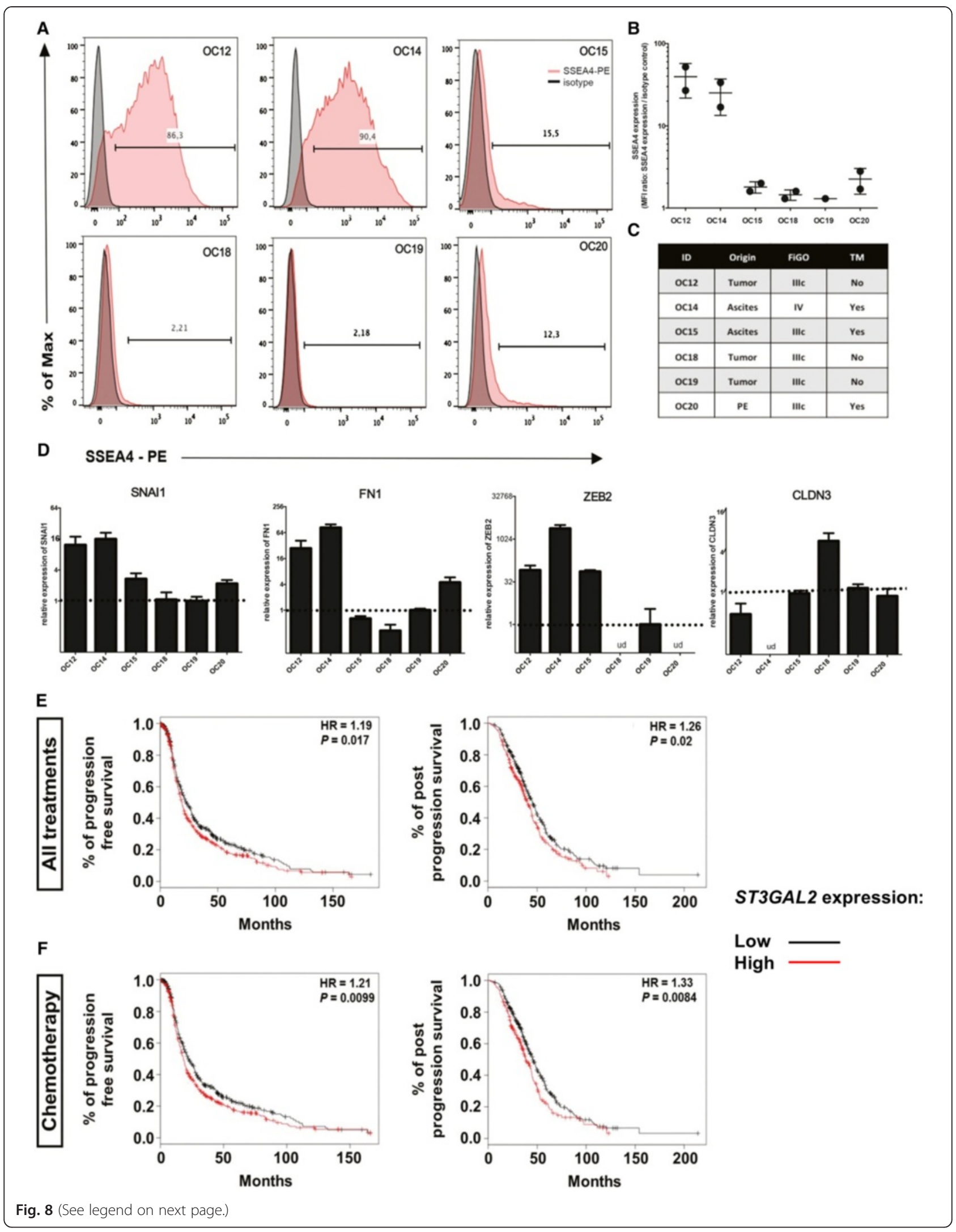


(See figure on previous page.)

Fig. 8 Sialyl-glycolipid stage-specific embryonic antigen 4 (SSEA4) and CMP-N-acetylneuraminate- $\beta$-galactosamide-a-2,3-sialyltransferase 2 (ST3GAL2) expression in ovarian cancer cells correlates with a mesenchymal phenotype and patient prognosis. a Flow cytometric analyses of SSEA4-phycoerythrin (red line) and respective isotype control (black line) on primary cells from patient-matched ovarian cancer cells. Numbers indicate percentage of positive cells compared with the isotype control. $\mathbf{b}$ Quantification of SSEA4 expression on six patient-matched primary ovarian cancer cell lines expressed as median fluorescence intensity (MFI) ratio. The MFI ratio is calculated as the MFI of SSEA4-stained cells divided by the MFI of the respective isotype control-stained cells. c Overview of patient-derived ovarian cancer sample characteristics. PE pleural effusion, TM treatment, FIGO International Federation of Gynecologists and Obstetricians. d Quantitative real-time polymerase chain reaction analysis of genes related to mesenchymal (SNAI1, FN1, ZEB2) and epithelial (CLDN3) phenotypes in the six patient-matched primary ovarian cancer cell lines. Error bars represent SD (performed in triplicates). ud undetected). e and f ST3GAL2 expression in ovarian cancer significantly correlates with poor prognosis (e), particularly in patients who underwent chemotherapy (f). HR hazard ratio

SSEA4-positive cell enrichment is a transient event, it is possible that the SSEA4-positive and SSEA4-negative cell balance tends to revert to the original ratio of positive and negative subpopulations observed in the untreated tumor during the latency period between cell injection and macroscopic tumor growth.

Tumors with initially high levels of SSEA4-positive cells seemed to be de novo resistant to chemotherapy. One important question is whether SSEA4 is upregulated during chemotherapy or if preexisting SSEA4positive cells are selected. The four TNBC PDXs used for the antibody screening had been established from untreated tumors and received a maximum of two cycles of chemotherapy, whereas four to six cycles are administered in the clinic. This might explain why PDXs from metastatic breast cancer, as well as primary cells from ovarian cancers, previously exposed to genotoxic therapy show very high initial percentages of SSEA4-positive cells. It is possible that this difference correlates with reversible or irreversible enrichment of the SSEA4 population.

Molecular analysis suggested a higher correlation of the drug resistance phenotype with the miRNA rather than the mRNA expression profile. Among mRNAs differentially expressed, a significant enrichment of genes involved in cellular import and export, response to toxins, and oxidative stress was observed, pathways that are connected to drug response at least on a global tumor level [32]. This is consistent with the reported role of globo series GSLs in MDR1 upregulation via the activation of cSrc signaling [33]. One striking observation was the downregulation of epithelial markers in conjunction with the overrepresentation of mesenchymal markers [34] at the mRNA level, and particularly, the concomitant downregulation of their regulatory miRNAs. Sustaining the observation that SSEA4-positive tumor cells show a more mesenchymal phenotype, we also found that induction of EMT enhances SSEA4 expression. This is in concordance with our observation that SSEA4 expression is found in metastatic cells that survived genotoxic chemotherapy. A large body of evidence connects EMT to drug resistance $[35,36]$ and metastasis $[37,38]$, providing a mechanistic explanation that might underlie the observed effects in SSEA4-positive tumor cells.

Two of the differentially regulated miRNAs-miR141 and miR-200a-have been shown to influence resistance to cisplatin and carboplatin in ovarian cancer by controlling the oxidative stress response [39]. We found that SSEA4 expression also correlates with a mesenchymal state and drug resistance in ovarian cancer.

Besides the EMT phenotype, the expression of SSEA4 is also likely regulated by miRNAs among different tumor subpopulations. The direct link between ST3GAL2 and SSEA4 was proven by siRNAmediated knockdown of ST3GAL2 resulting in a significant decrease of SSEA4 expression and by positive correlation between SSEA4 and ST3GAL2 expression in PDX models. Interestingly, all five miRNAs predicted to target ST3GAL2 are also directly involved in EMT and drug resistance $[39,40]$. Even if further studies clarify whether ST3GAL2 is directly involved in drug resistance, this regulatory mechanism, in combination with the overexpression of resistance-associated genes, such as transporters of the MDR family, might be the underlying mechanisms linking EMT and drug resistance to SSEA4 expression.

\section{Conclusions}

We have identified SSEA4 to mark a subpopulation of chemotherapy-resistant, mesenchymal breast cancer cells. Furthermore, we have shown that the expression level of ST3GAL2, the enzyme catalyzing SSEA4 synthesis, can be used as a marker to predict clinical outcome of breast and ovarian cancer patients, in particular those treated with chemotherapy. SSEA4 and ST3GAL2 may therefore represent key markers to classify patient groups in order to avoid ineffective and painful therapies and to develop alternative treatment regimens for breast cancer patients. 


\section{Additional files}

Additional file 1: Supplemental experimental procedures. Detailed description of materials and methods. (DOCX $62 \mathrm{~kb}$ )

Additional file 2: Table S1. Antibodies used for the screening approach. Detailed description of used antibodies. (DOCX $46 \mathrm{~kb}$ )

Additional file 3: Figure S1. Representative gating strategy for flow cytometry-based marker analysis of dissociated xenograft tumor tissue. Tumor tissue was dissociated to obtain a single-cell suspension while preserving cell surface epitopes. The sample was stained for mouse-specific markers to exclude cells of murine origin from the analysis as well as for the screening candidates and analyzed by multiparametric flow cytometry. Doublets were excluded by forward scatter (FSC) area/FSC height gating (a); debris was excluded by FSC/side scatter gating (b); dead cells were excluded by gating off propidium iodide-positive events (c) and mouse cells were excluded by gating on a-mouse-fluorescein isothiocyanate-negative events (d). When we screened two samples in parallel, we found that one of the samples was labeled using an ultraviolet dye, allowing for subsequent separation of the events of each sample by gating on the VioBlue channel fluorescence intensity (e-h). (PNG 736 kb)

Additional file 4: Figure S2. Anti-SSEA4 antibodies derived from clone REA101 and MC-813-70 recognize the same epitope. Flow cytometric analysis of an antibody cross-blocking experiment on human induced pluripotent stem cells. Cells were either directly fluorescently labeled using anti-SSEA-4-phycoerythrin conjugates from clone REA101 (a) or MC-813-70 (b) or after cells had been blocked by preincubation with $100 \mathrm{\mu g} / \mathrm{ml}$ unconjugated antibody of the alternative clone $(\mathbf{a}, \mathbf{b})$. The fluorescent labeling of the REA101-derived anti-SSEA-4-phycoerythrin antibody was strongly diminished by blocking with an excess of MC-813-70 unconjugated antibody (a), while unconjugated REA101 caused a complete block of the fluorescent labeling of MC-813-70derived anti-SSEA-4-phycoerythrin antibody (b). These results indicate that both antibodies recognize the same epitope and that the REA101-derived antibody has a higher functional affinity than the one derived from clone MC-813-70. (PNG $107 \mathrm{~kb}$ )

Additional file 5: Figure S3. Correlation among CD24-, CD44-, and SSEA4-expressing subpopulations. To address the correlation between CD24, CD44, and SSEA4 expression, we performed costaining of these markers on residual tumor nodules after AC chemotherapy and untreated tumors of three independent models: $\mathrm{HBCx}-6$ (a), $\mathrm{HBCx}-10$ (b), and $\mathrm{HBC}-14$ (c). Regulation of the three markers did not correlate among the treatment cycles. (PNG $1664 \mathrm{~kb}$ )

Additional file 6: Figure S4. Expression of SSEA4 in tumors responsive or resistant to chemotherapeutic treatment. Tumors responsive $(n=6)$ or resistant $(n=4)$ to AC treatment were analyzed for expression of SSEA4. Three of the four resistant tumor models showed higher percentages of SSEA4-positive cells than all of the six responsive tumors. In two of the resistant tumor models, almost all of the cells expressed SSEA4. (TIFF $61 \mathrm{~kb}$ )

Additional file 7: Figure S5. Tumor-initiating capacity of the SSEA4positive and SSEA4-negative subpopulation. (a) One hundred thousand freshly dissociated SSEA4-positive or SSEA4-negative cells were injected in two groups of eight mice each. Tumor volume was measured once per week, and the mean volume of both groups was calculated. The significance level ( $p$ value by $t$ test) is indicated above each time point. (b) The frequency of SSEA4-expressing cells in the parental tumor model HBCX-14 and in tumors that originated from the SSEA4positive or SSEA4-negative subpopulation was determined by flow cytometry, which indicated a regulation of SSEA4 expression back to the initial level during the growth phase in vivo. (PNG 93 kb)

Additional file 8: Table S2. Treatment history of breast cancer patients. Detailed description of treatment history of breast cancer patients. (XLSX $11 \mathrm{~kb}$ )

Additional file 9: Table S3. Results of microarray analysis. Results of gene and miRNA expression profiling, Gene Ontology analysis, and miRNA target prediction. (XLSX $118 \mathrm{~kb}$ )

Additional file 10: Figure S6. SSEA4-positive breast cancer cells show differential expression of genes pointing toward a mesenchymal state as well as increased expression of members of the SLC and multidrug resistance ATP-binding cassette transporter families, but not of stem cell associated transcripts. (a) In the SSEA4-positive cell fraction, genes characteristic of an epithelial state showed decreased expression compared with the SSEA4-negative fraction. In contrast, genes characteristic of a mesenchymal state showed increased expression compared with the SSEA4-negative fraction. (b) In the SSEA4-positive cell fraction, members of the SLC and multidrug resistance ATP-binding cassette transporter families showed increased expression compared with the SSEA4-negative fraction. (c) Stem cell markers were not consistently regulated among the SSEA4positive and SSEA4-negative cell fractions. The housekeeping gene GAPDH showed no significant regulation among the subpopulations. Each bar represents the $\log _{2}$ expression ratio of the SSEA4-positive fraction relative to the SSEA4-negative fraction for the respective tumor model. (TIFF $655 \mathrm{~kb}$ )

Additional file 11: Figure S7. Validation of miRNA candidates using a flow cytometry-based 39-plex miRNA assay. (a) Cluster analysis of expression ratios ( $\log _{2}$-transformed) obtained from hybridization of SSEA4-positive (pos) and SSEA4-negative (neg) samples. The miRNAs that were significantly downregulated in SSEA4-positive cells based on the microarray analysis are highlighted with a blue bar. (b) Comparison of miRNA bead assay (BA) and microarray data (MA; average of all three samples). Seven miRNAs that were differentially expressed between SSEA4-positive and SSEA4-negative cells, as well as three miRNAs (miR-30b-5p, miR-29a-3p, and miR-16-5p) expressed at a similar level in both cell types, are shown. The bead assay results correlated well with the microarray data. (PNG 509 kb)

Additional file 12: Figure S8. SSEA4-positive breast cancer cells show decreased expression of miRNAs inhibiting EMT inducers. Expression ratios of the 12 miRNAs targeting the key mesenchymal regulator and indicator genes ZEB1, ZEB2, fibronectin 1, Snail1, Snail2, and Twist. Each bar represents the $\log _{2}$ expression ratio of the SSEA4-positive fraction relative to the SSEA4-negative fraction for the respective tumor model. (TIFF 201 kb)

Additional file 13: Figure S9. Expression of SSEA4 in RCC and healthy kidney tissue. Primary RCC and healthy kidney tissues from the same patient were dissociated and analyzed by multiparametric flow cytometry. Doublets were excluded by FSC-A/FSC-H gating (a); debris was excluded by FSC/SSC gating (b); dead cells were excluded by gating off $\mathrm{PI}^{+}$events (c); and lineage-positive cells were excluded by gating on a-Lin-FITC-negative events (d). In each patient, healthy and tumor tissues were analyzed in parallel in one labeling reaction. Therefore, one of the samples was labeled using a UV dye, allowing for subsequent separation of the events of each sample by gating on the VioBlue channel fluorescence intensity $(\mathbf{e}-\mathbf{h})$. In all of the analyzed patients $(n=3)$, the expression of SSEA4 was strongly increased in the tumor tissue as compared with the respective healthy tissue, with almost all tumor cells expressing SSEA4 in two of the patients $(\mathbf{f}-\mathbf{h})$. ${ }^{*} \mathrm{a}-$ Lin-FITC = CD45-FITC, CD31-FITC, CD235a (glycophorin A)-FITC. (PNG $717 \mathrm{~kb}$ )

\section{Abbreviations}

AC: doxorubicin/cyclophosphamide; APC: allophycocyanin; CT: chemotherapy; CTC: circulating tumor cell; DAPI: 4',6-diamidino-2-phenylindole; EMT: epithelialmesenchymal transition; EpCAM: epithelial cell adhesion molecule; ER: estrogen receptor; FIGO: International Federation of Gynecologists and Obstetricians; FITC: fluorescein isothiocyanate; FSC: forward scatter; FSC-A: forward scatter area; FSC-H: forward scatter height; GEO: Gene Expression Omnibus; GSL: glycosphingolipid; HBCx: human breast cancer xenograft; $I C_{50}$ : halfmaximal inhibitory concentration; MFI: median fluorescence intensity; miRNA: microRNA, mIR; mRA: messenger RNA; PDX: patient-derived xenograft; PE: pleural effusion; PI: propidium iodide; PR: progesterone receptor; RCC: renal cell carcinoma; siRNA: small interfering RNA; SSC: side scatter; SSEA4: sialyl-glycolipid stage-specific embryonic antigen 4; ST3GAL2: CMP-N-acetylneuraminate- $\beta$-galactosamide- -2 -2,3-sialyltransferase 2; TGF: transforming growth factor; TM: treatment; TNBC: triple-negative breast cancer. 


\section{Competing interests}

$A A, S T, U B, S K, S R, B G, D A, A B$, and $O H$ are or were full-time employees of Miltenyi Biotec $\mathrm{GmbH}$. EP, OD, JGJ, and SC are or were full time employees of XenTech SAS. The other authors declare that have no competing interests.

\section{Authors' contributions}

$A A, E P, U B, M S, S K, M R S, A T r, J G J, A B, S C$, and $O H$ conceived and designed the experiments. AA, EP, OD, UB, MSa, FMZ, SK, SW, MR, and DA performed the experiments. AA, EP, ST, UB, OD, MSa, FMZ, SR, BG, SK, MRS, ATr, JGJ, AB, SC, and $\mathrm{OH}$ analyzed the data. SS, MSü, AS, ATe, MF, and LS provided crucia samples and reagents. $A A, E P, A B, S C$, and $O H$ wrote the manuscript draft. All authors were involved in revising the manuscript critically for important intellectual content. All authors read and approved the final manuscript and agree to be accountable for all aspects of the work in ensuring that questions related to the accuracy or integrity of any part of the work are appropriately investigated and resolved.

\section{Acknowledgments}

We are grateful to Jana Ciomperlik, Janina Kuhl, Nadine Chelius, Petra Kussmann, Tanja Töpfer, Jens Gaiser, Alena Böttcher, Sophie Banis, Erwan Selingue, and Vanessa Yvonnet for excellent technical assistance. Furthermore, we thank Yvonne Diener for supplying us with the CD133 siRNAs and for support in designing the knockdown experiments. In addition, we are grateful to Michail Knauel for help with the microarray data analysis. This work was supported by the Eurocancer Stem Cell Training Network (EuroCSCTraining) of the European Union (Innovative Training Networks FP7 Program; Marie Skłodowska-Curie Actions grant 264361) and by the Dietmar Hopp Foundation (to ATr).

\section{Author details}

'Miltenyi Biotec GmbH, Friedrich-Ebert-Strasse 68, 51429 Bergisch Gladbach, Germany. ${ }^{2}$ XenTech SAS, 4 rue Pierre Fontaine, 91000 Evry, France. ${ }^{3}$ Present address: Department of Virology, Pasteur Institute, 25-28 Rue du Docteur Roux, 75015 Paris, France. ${ }^{4}$ Heidelberg Institute for Stem Cell Technology and Experimental Medicine (HI-STEM) gGmbH, Im Neuenheimer Feld 280, 69120 Heidelberg, Germany. ${ }^{5}$ Division of Stem Cells and Cancer, German Cancer Research Center (DKFZ), Im Neuenheimer Feld 280, 69120 Heidelberg, Germany. ${ }^{6}$ Frauenklinik, University Medical Centre Mannheim, Theodor-Kutzer-Ufer 1-3, 68167 Mannheim, Germany. ${ }^{7}$ National Center for Tumor Diseases, University Hospital Heidelberg, Im Neuenheimer Feld 460, 69120 Heidelberg, Germany. ${ }^{8}$ Sidra Medical and Research Center, PO Box 26999, Doha, Qatar. ${ }^{9}$ Department of Health Sciences, University of Milan, Via Festa del Perdono 7, 20122 Milan, Italy. ${ }^{10}$ German Cancer Consortium, Im Neuenheimer Feld 280, 69120 Heidelberg, Germany. ${ }^{11}$ University of Ferrara, LTTA Centre,Department of Morphology, Surgery and Experimental Medicine, Via Fossato di Mortara 70, 44121 Ferrara, Italy.

\section{Received: 1 April 2015 Accepted: 16 September 2015}

Published online: 25 November 2015

\section{References}

1. Rivenbark AG, O'Connor SM, Coleman WB. Molecular and cellular heterogeneity in breast cancer: challenges for personalized medicine. Am J Pathol. 2013;183(4):1113-24.

2. Ades F, Zardavas D, Bozovic-Spasojevic I, Pugliano L, Fumagalli D, de Azambuja E, et al. Luminal B breast cancer: molecular characterization, clinical management, and future perspectives. J Clin Oncol. 2014;32(25):2794-803.

3. Irvin Jr WJ, Carey LA. What is triple-negative breast cancer? Eur J Cancer. 2008:44(18):2799-805.

4. Davis SL, Eckhardt SG, Tentler JJ, Diamond JR. Triple-negative breast cancer: bridging the gap from cancer genomics to predictive biomarkers. Ther Adv Med Oncol. 2014;6(3):88-100.

5. Liedtke C, Mazouni C, Hess KR, Andre F, Tordai A, Mejia JA, et al. Response to neoadjuvant therapy and long-term survival in patients with triplenegative breast cancer. J Clin Oncol. 2008;26(8):1275-81.

6. Weigelt B, Pusztai L, Ashworth A, Reis-Filho JS. Challenges translating breast cancer gene signatures into the clinic. Nat Rev. 2011;9(1):58-64.

7. Hardt O, Wild S, Oerlecke I, Hofmann K, Luo S, Wiencek Y, et al. Highly sensitive profiling of $\mathrm{CD} 44^{+} / \mathrm{CD} 24^{-}$breast cancer stem cells by combining global mRNA amplification and next generation sequencing: evidence for a hyperactive PI3K pathway. Cancer Lett. 2012;325(2):165-74.

8. Sabbah M, Emami S, Redeuilh G, Julien S, Prévost G, Zimber A, et al Molecular signature and therapeutic perspective of the epithelial-tomesenchymal transitions in epithelial cancers. Drug Resist Updat. 2008;11(4-5):123-51.

9. Marangoni E, Vincent-Salomon A, Auger N, Degeorges A, Assayag F, de Cremoux $P$, et al. A new model of patient tumor-derived breast cancer xenografts for preclinical assays. Clin Cancer Res. 2007;13(13): 3989-98.

10. DeRose YS, Wang G, Lin YC, Bernard PS, Buys SS, Ebbert MT, et al. Tumor grafts derived from women with breast cancer authentically reflect tumor pathology, growth, metastasis and disease outcomes. Nat Med. 2011;17(11):1514-20.

11. Bradbury A, Pluckthun A. Reproducibility: standardize antibodies used in research. Nature. 2015;518(7537):27-9.

12. Bradbury AM, Pluckthun A. Antibodies: validate recombinants once. Nature. 2015;520(7547):295

13. Barrett T, Wilhite SE, Ledoux P, Evangelista C, Kim IF, Tomashevsky M, et al. NCBI GEO: archive for functional genomics data sets-update. Nucleic Acids Res. 2013;41(Database issue):D991-5.

14. Edgar R, Domrachev M, Lash AE. Gene Expression Omnibus: NCBI gene expression and hybridization array data repository. Nucleic Acids Res. 2002;30(1):207-10

15. Györffy B, Lanczky A, Eklund AC, Denkert C, Budczies J, Li Q, et al. An online survival analysis tool to rapidly assess the effect of 22,277 genes on breast cancer prognosis using microarray data of 1,809 patients. Breast Cancer Res Treat. 2010;123(3):725-31.

16. Györffy B, Lánczky A, Szállási Z. Implementing an online tool for genome-wide validation of survival-associated biomarkers in ovariancancer using microarray data from 1287 patients. Endocr Relat Cancer. 2012;19(2):197-208

17. Trumpp A, Wiestler OD. Mechanisms of disease: cancer stem cells_-targeting the evil twin. Nat Clin Pract. 2008;5(6):337-47.

18. Prowell TM, Pazdur R. Pathological complete response and accelerated drug approval in early breast cancer. N Engl J Med. 2012;366(26):2438-41.

19. Baccelli I, Schneeweiss A, Riethdorf S, Stenzinger A, Schillert A, Vogel V, et al. Identification of a population of blood circulating tumor cells from breast cancer patients that initiates metastasis in a xenograft assay. Nat Biotechnol. 2013;31(6):539-44

20. Wong SQ, Li J, Salemi R, Sheppard KE, Do H, Tothill RW, et al. Targetedcapture massively-parallel sequencing enables robust detection of clinically informative mutations from formalin-fixed tumours. Sci Rep. 2013;3:3494

21. Ashburner M, Ball CA, Blake JA, Botstein D, Butler H, Cherry JM, et al. Gene Ontology: tool for the unification of biology. Nat Genet. 2000;25(1):25-9.

22. Saito S, Aoki H, Ito A, Ueno S, Wada T, Mitsuzuka K, et al. Human a2,3sialyltransferase (ST3Gal II) is a stage-specific embryonic antigen-4 synthase. J Biol Chem. 2003;278(29):26474-9.

23. Hegde GV, de la Cruz C, Eastham-Anderson J, Zheng Y, Sweet-Cordero EA, Jackson EL. Residual tumor cells that drive disease relapse after chemotherapy do not have enhanced tumor initiating capacity. PLoS One. 2012;7(10), e45647.

24. D'Angelo RC, Liu XW, Najy AJ, Jung YS, Won J, Chai KX, et al. TIMP-1 via TWIST1 induces EMT phenotypes in human breast epithelial cells. Mol Cancer Res. 2014;12(9):1324-33.

25. Cohen HT, McGovern FJ. Renal-cell carcinoma. N Engl J Med. 2005:353(23):2477-90.

26. McGuire 3rd WP, Markman M. Primary ovarian cancer chemotherapy: current standards of care. Br J Cancer. 2003:89 Suppl 3:S3-8.

27. Al-Hajj M, Wicha MS, Benito-Hernandez A, Morrison SJ, Clarke MF. Prospective identification of tumorigenic breast cancer cells. Proc Natl Acad Sci U S A. 2003;100(7):3983-8

28. Ricci-Vitiani L, Lombardi DG, Pilozzi E, Biffoni M, Todaro M, Peschle C, et al. Identification and expansion of human colon-cancer-initiating cells. Nature. 2007:445(7123):111-5.

29. Zhang S, Balch C, Chan MW, Lai HC, Matei D, Schilder JM, et al. Identification and characterization of ovarian cancer-initiating cells from primary human tumors. Cancer Res. 2008;68(11):4311-20.

30. Liu YY, Hill RA, Li YT. Ceramide glycosylation catalyzed by glucosylceramide synthase and cancer drug resistance. Adv Cancer Res. 2013;117:59-89. 
31. Maugeri-Sacca M, Vigneri $P$, De Maria R. Cancer stem cells and chemosensitivity. Clin Cancer Res. 2011;17(15):4942-7.

32. Zinzi L, Capparelli E, Cantore M, Contino M, Leopoldo M, Colabufo NA. Small and innovative molecules as new strategy to revert MDR. Front Oncol. 2014;4:2.

33. Liu YY, Gupta V, Patwardhan GA, Bhinge K, Zhao Y, Bao J, et al Glucosylceramide synthase upregulates MDR1 expression in the regulation of cancer drug resistance through $\mathrm{cSrc}$ and $\beta$-catenin signaling. Mol Cancer. 2010;9:145.

34. Lee JM, Dedhar S, Kalluri R, Thompson EW. The epithelial-mesenchymal transition: new insights in signaling, development, and disease. J Cell Biol. 2006;172(7):973-81.

35. Shang $Y$, Cai $X$, Fan D. Roles of epithelial-mesenchymal transition in cancer drug resistance. Curr Cancer Drug Targets. 2013;13(9):915-29.

36. Siebzehnrub| FA, Silver DJ, Tugertimur B, Deleyrolle LP, Siebzehnrubl D, Sarkisian MR, et al. The ZEB1 pathway links glioblastoma initiation, invasion and chemoresistance. EMBO Mol Med. 2013;5(8):1196-212.

37. Brabletz T. EMT and MET in metastasis: where are the cancer stem cells? Cancer Cell. 2012;22(6):699-701.

38. Yang Y, Ahn YH, Gibbons DL, Zang Y, Lin W, Thilaganathan N, et al. The Notch ligand Jagged2 promotes lung adenocarcinoma metastasis through a miR-200-dependent pathway in mice. J Clin Invest. 2011;121(4):1373-85.

39. Mateescu B, Batista L, Cardon M, Gruosso T, de Feraudy Y, Mariani O, et al. miR-141 and miR-200a act on ovarian tumorigenesis by controlling oxidative stress response. Nat Med. 2011;17(12):1627-35.

40. Polyak K, Weinberg RA. Transitions between epithelial and mesenchymal states: acquisition of malignant and stem cell traits. Nat Rev Cancer. 2009:9(4):265-73.

\section{Submit your next manuscript to BioMed Central and take full advantage of:}

- Convenient online submission

- Thorough peer review

- No space constraints or color figure charges

- Immediate publication on acceptance

- Inclusion in PubMed, CAS, Scopus and Google Scholar

- Research which is freely available for redistribution 